\title{
Biomarkers Through The Development, Progression and Chronic Complications of Diabetes Mellitus: A Mini-Review
}

\author{
Maddy Narváez-Méndez , Samantha Morgan², Martín Coronado-Malagón ${ }^{1}$, César Alejandro Arce-Salinas \\ Antonio Barajas ${ }^{3}$, Isabel Arenas ${ }^{3}$, Alejandro Svarch ${ }^{1,3}$ and David E. Garcia ${ }^{3 *}$ \\ ${ }^{1}$ Department of Internal Medicine, South Central Hospital of High Specialty, C.P. 14140, Mexico City, Mexico \\ ${ }^{2}$ College of Engineering, Georgia Institute of Technology, North Avenue, Atlanta, GA 30332, U.S.A \\ ${ }^{3}$ Department of Physiology, School of Medicine, Universidad Nacional Autónoma de México, C.P. 04510, Mexico City, Mexico
}

Received: November 01,2018; Accepted: November 19,2018; Published: : November 28,2018

*Corresponding author : David E. Garcia, Department of Physiology, School of Medicine, Universidad Nacional Autónoma de México, C.P. 04510, Mexico City, Mexico Tel: (52 55) 5623 2137;Fax: (52 55) 5623 2241;E-mail: erasmo@unam.mx

\begin{abstract}
Biomarkers are widely known to be elevated in patients with obesity, diabetes and related chronic complications such as nephropathy, retinopathy and cardiovascular disease. Obesity epidemic has resulted in an increased risk of diabetes development and consequent cardiovascular mortality, leading to probing the usefulness of the inflammatory biomarkers triggered by adipose tissue in individuals at risk. However, current knowledge regarding the clinical usefulness of inflammatory markers in individuals progressing from one stage to another is inconclusive. Therefore, the purpose of this review is to provide an insight on the clinical utility of temporary relationships of biomarker elevations at specific stages of type 2 diabetes. Recently it has been proposed that increased levels of low-grade inflammation biomarkers such as EN-RAGE and IL-13, may serve as indicators for risk of progression from normoglycemia to pre-diabetes and from pre-diabetes to diabetes, respectively. In the development of chronic complications of type 2 diabetes, biomarkers such as TNF-alpha have been proposed to serve as markers of incident albuminuria. Cardiac biomarkers may serve as feasible predictors of the risk of cardiovascular outcomes in diabetic patients. Furthermore, relationships amongst specific biomarkers have been proposed to function as possible future instruments of detection of T2DM such as IL-6, CRP and insulin. Clinicians may be benefited from biomarker profiles on the progression of the disease, minimizing economic and social repercussions.
\end{abstract}

Keywords: Inflammation; Obesity; Biomarkers; Insulin Resistance; Cardiac Biomarkers

\section{Introduction}

A wealth of information regarding the elevation of inflammatory biomarkers in severe diseases has led clinicians to inquiry about its clinical usefulness in the development of metabolic diseases such as type 2 Diabetes Mellitus (T2DM). In principle, various inflammatory markers have been associated with progression from normoglycemia to pre-diabetes (Interleukin-13, IL-13, extracellular newly identified receptor for advanced glycation end-products binding protein, EN-RAGE, and C Reactive Protein, CRP). However, only higher levels of a limited number of biomarkers of low-grade inflammation have been associated with the early progression of recently diagnosed diabetes [1]. The aim of this mini-review is to provide an insight on the clinical utility of temporary relationships of biomarker elevations at specific stages of T2DM. As an outstanding example, EN-RAGE, also known as S100A12 or Calgranulin C, has been wished for a promising inflammatory marker for pre-diabetes [2]. A general belief is that increased levels of EN-RAGE may serve as indicators for risk of progression from normoglycemia to prediabetes. Interestingly, high EN-RAGE levels have been associated with an increased risk of incident pre-diabetes, while the opposite has been thought for high levels of IL-13 [3]. Therefore, we examine the behavior of biomarkers as a potential indicator of the development, progression and chronic complications of T2DM.

\section{Biomarkers of T2DM risk factors}

Obesity is one of the main risk factors for the development of T2DM. White adipose tissue (WAT) is known to have an active role performing many metabolic and endocrine functions in the body [4]. Some of these endocrine functions include the secretion of bioactive proteins (adipocytokines), interleukin-6 (IL-6), tumor necrosis factor alpha (TNF-alpha), resistin, free fatty acids (FFA) and plasminogen activator inhibitor 1 (PAI-1) [5]. The expression of these molecules is related to body weight, increasing with fat accumulation. As a result, a pro-inflammatory state is created within obese WAT [5].

Two types of macrophage cytokine secretion profiles compete within the WAT: the M1 and M2 profiles [5]. The M1 profile is pro-inflammatory and promotes insulin insensitivity, while M2 produces anti-inflammatory markers [5]. The WAT in the obese population produces low-grade inflammatory markers such as IL-6, while the WAT of lean people produces adiponectin [5]. Adiponectin is an anti-inflammatory protein that naturally inhibits TNF-alpha, increases Interleukin-1 receptor antagonist 


\section{Biomarkers Through The Development, Progression and Chronic Complications of Diabetes Mellitus: A Mini-Review}

(IL-1Ra) and thus promotes anti-apoptosis of the beta cells in pancreatic islets and insulin sensitivity [6]. It has been proved that adiponectin levels maintain an inverse relationship with visceral fat and its levels are decreased in obese diabetics and patients with heart disease [6]. Thus, the WAT can tip the balance towards either an M1 or M2 profile through the secretion of IL-6, which explains the anti-inflammatory environment of the adipose tissue of lean populations contrasting with the inflammatory environment of the obese population [5]. Immune cells are further recruited to the tissues through chemotactic signaling that are increased in WAT of obese patients. One such signaling molecule is monocyte chemotactic protein 1 (MCP-1), secreted by adipocytes, which regulates monocyte trafficking into the tissues [7].

Besides the macrophages, other immune cells are present in the WAT, particularly lymphocytes, which can also have different profiles such as Th1, Th2 and Th17 [8]. For example, the $\mathrm{T}$ regulatory lymphocytes are decreased in obese individuals [8]. Their modulation of the immune system is through antiinflammatory molecules: interleukin-10 (IL-10), interleukin-33 (IL-33), peroxisome proliferator-activated receptor gamma (PPAR gamma), that encourage the maturation of M2 macrophages [8]. Lymphocytes can also promote the maintenance of a proinflammatory profile [8]. Some interleukin pro-inflammatory mediators (Interleukin-17A, IL-17A, and Interleukin-17F, IL$17 \mathrm{~F}$ ), present in autoimmune inflammatory diseases such as Crohn disease, multiple sclerosis and rheumatoid arthritis [9], are also related to the development of glucose intolerance and the development of chronic complications of T2DM. Some authors have reported high levels of IL-17 in patients with recently diagnosed type 2 diabetes [10]; others have described an inversely proportional relationship between levels of IL-17 and the presence of T2DM retinopathy [11]. Additional studies should be addressed to work out this point.

Obesity, inflammation and increased insulin resistance are associated with the production of IL-17, increased secretion of TNF-alpha, interleukin-1 beta (IL-1beta), providing positive feedback to M1 macrophages [8], ensuring the continuity of this metabolic imbalance. In the long term, the perpetuation of this inflammatory state eventually results in impaired triglyceride deposition and an increase in circulating FFA [8]. This affects oxidative phosphorylation, concluding in impaired insulinmediated glucose-uptake, thus creating insulin resistance [12].

Tissues under stress generated by insulin resistance respond by secreting chemokines like MCP-1 and macrophage migration inhibition factor [13]. This, in turn, increases the expression of adhesion molecules such as interstitial and vascular cellular adhesion molecules (ICAM-1, VCAM-1) and E-selectin, crucial for recruitment and diapedesis of immune cells [14, 15]. This process continues the pro-inflammatory state by further activation of cytokines like TNF-alpha, interleuin-1 (IL-1), IL-6 and interleukin-18 (IL-18) [14] that concludes in endothelial dysfunction. The vascular stress response also promotes the production of ICAM-1, whose levels increase secundarily to inflammatory cytokines [16].
These processes accelerate the production of PAI-1, tissue factor, fibrinogen and factor VIII, thus generating a procoagulant state [12]. PAI-1, is one of the earliest detectable biomarkers of insulin resistance, as is not only associated with endothelial function but also with proatherosclerotic and prothrombic changes [14]. Elevations of this factor correlate with diabetes onset independent of direct measurements of insulin resistance and obesity. However, as glucose intolerance develops into prediabetes it becomes unspecific, suggesting to be most useful for very early stages of detection [14].

Tissular response to stress increases the levels of CRP, a major acute-phase response mediator induced by IL-6 [15]. As a biomarker of hyperglycemia, CRP is considered a strong indicator of uncontrolled diabetes [17]. It has been associated with endothelial activation [18], correlating with microalbuminuria, increasing the risk of coronary heart disease, myocardial infarction and ischemic stroke $[1,19]$.

The development of glucose impairment is related to the production of some pro-inflammatory interleukins, particularly IL-1 beta and IL-6 [18, 20, 21]. Increased levels of IL-1 beta promote insulin insensitivity and impairment of $\beta$-cell function, conferring risk of development T2DM [21].IL-6, a crucial marker in the innate immune response to T2DM [18] induces inflammation through the hepatic biosynthesis of CRP [18]. Elevated levels of IL- 6 are also associated with increases in impaired glucose tolerance (IGT), insulin resistance and increased production of acute-phase proteins [20]. The combination of adjusted IL-6 levels for body mass index and fasting insulin with CRP levels have a multiplicative effect on detection of T2DM [18].

\section{Biomarkers of T2DM onset}

\section{Glycation Biomarkers}

To emphasize the usefulness of early detection, the further study of phase-specific associations has increased. It has been published that increased levels of EN-RAGE correlated with higher risk of incident pre-diabetes while increased levels of IL-13 decreased the risk of incident pre-diabetes, diabetes and insulinneed. [3]. Additionally, increased levels of ICAM and VCAM were found to be independent risk factors of faster progression from normoglycemia to T2DM [22].

Mast cells, dendritic cells, eosinophils, and Th2 lymphocytes [23] produce IL-13, which down-regulates the body's immunodiabetogenic pathways [23]. In animal models, IL-13 regulates glucose homeostasis in the liver, proposing it to be a key target in future research [24].

EN-RAGE, a product of granulocytes with pro-inflammatory properties, has also been postulated to serve as a marker for incident coronary heart disease, independently of CPR [3].

\section{Endothelial Dysfunction Biomarkers}

It is commonly held that endothelial dysfunction and low-grade inflammation can explain, at least in part, why deteriorating glucose tolerance is associated with cardiovascular disease $[19,25]$. However there is no direct consistent evidence 
to prove it [26]. The identification of biomarkers signaling lowgrade inflammation and endothelial dysfunction preceding atherosclerosis and development of $\mathrm{T} 2 \mathrm{DM}$, represent an opportunity for clinicians to identify individuals at risk [3, $19,25]$. Biomarkers could serve as endothelial dysfunction indicators and independently predict the risk of future T2DM [3, 19]. An increase in levels of these molecules can also be found in patients with hypertension and pre-diabetes, possibly translating as higher risk for cardiovascular complications [14] as they have been described as a risk factor for coronary heart disease [27]. Specifically, the combination of high ICAM and VCAM values is considered the strongest predictor of cardiovascular risk in diabetic patients [15].

In a population-based study, impaired glucose metabolism was associated only with low-grade inflammation, whereas T2DM was independently associated with both endothelial dysfunction and low-grade inflammation, resulting in increased risk of cardiovascular mortality [27]. Specifically, endothelial dysfunction associated with low-grade inflammation in T2DM was reported to account for $43 \%$ increase in cardiovascular mortality risk [27].

Another adhesion molecule, E- selectin, is now considered a marker of endothelial dysfunction and is considered as a strong indicator for cardiovascular disease [16]. Involved in leucocyte rolling along the endothelium, activated E-selectin is released into the blood stream where it modulates blood pressure [16] and glucose-stimulated insulin secretion [36]. Increased levels of E-selectin are associated with myocardial infarctions as well [16] which suggests the importance of this biomarker at later stages.

\section{Biomarkers of T2DM complications}

\section{Microvascular T2DM complications}

Microvascular disease in T2DM can target different organs, each one with their own particularities. We will review the described biomarkers for kidney and retina damage.

Increased levels of TNF-alpha, an immune cell regulator and cell-signaling protein mainly secreted by adipocytes, are present in diabetics with micro or macro albuminuria [28]. Authors have reported that the expression of ICAM reflects the progression of diabetic nephropathy [29], however, some others described that patients with T2DM with microvascular complications have lower levels of ICAM compared to healthy age-matched subjects [30]. Another adhesion molecule, VCAM-1, has been connected to microvascular complications of T2DM. Increased levels of VCAM-1 are often associated with albuminuria [29]. Studies have discussed the relationship that these 2 molecules have with the progression of diabetic nephropathy and concluded that VCAM appears to be more specific compared to ICAM, which only portrays systemic inflammation. [31].

Diabetic Retinopathy (DR) is a common, important, and preventable cause of blindness usually diagnosed in the later stages of the disease. Increased levels of ICAM have been proposed to be markers in early stages of DR [30]. Another marker, adiponectin, was demonstrated to be lower in patients with DR [32]. Research is being conducted to find biomarkers for early detection of DR, which include MCP-1, Cathepsin-D [13] and adhesion molecules such as E-selectin. The results of the studies concerning this last marker and DR are inconclusive about its role [33] and further research is needed. Specifically, cathepsin-D, a metabolic protease, is a known biomarker for cardiovascular disease (CVD) diagnosis in patients with chronic kidney disease and it has been correlated with insulin resistance $[13,34]$. Increased levels of Cathepsin-D have been found in serum samples of patients with DR [13], especially in youngonset cases, leading researchers to consider it a vascular ager, along with MCP-1 [13]. Increased levels of MCP-1 were initially associated with DR [13] however; this association is attenuated by traditional risk adjustments such as lifestyle or clinical factors [13].

\section{Macrovascular T2DM complications}

The large amount of literature regarding the use of cardiac biomarkers in T2DM detection has accentuated the need of discussing the clinical utility of markers of cardiac biochemical stress, vascular remodeling and low-grade inflammation, such as natriuretic peptides (NPs), galectin-3 (Gal-3), interleukins and growth differentiation factor-15 (GDF-15) [35, 36, 37]. These biomarkers have been proposed as future candidates of diagnostic or prognostic value in diabetic patients [37].

Secretion of NPs results from volume overload or the stretching of the cardiac wall [36]. However, recent studies have shown that the NP system function is deficient in obese and diabetic patients due to worse clearance of NP receptors and neutral endopeptidases [36]. Thus, their role in T2DM progression appears to be strongly controversial. Consequently, the NP system in obese and diabetic patients is not able to mediate a wide spectrum of cardiovascular and metabolic protective effects that involve mechanisms of vasodilation, natriuresis, lipolysis, weight loss, lusitropy, lipid peroxidation, mitochondrial respiration improvement and insulin sensitivity [37]. However, according to contemporary clinical guidelines for heart failure, NPs are recognized as powerful biomarkers for acute coronary syndrome and cardiac cytotoxicity, and they provide more accurate diagnostic and predictive values $[38,39]$. Thus, isolated obesity remains the crucial condition for decision-making regarding predictive role of NPs and requires more scrutiny [44]. Given all these limitations and controversies, NPs are proposed diagnostic biomarkers linking T2DM with cardiovascular (CV) risk and CV outcomes [37].

Gal-3 is a lectin that belongs to soluble beta-galactosidebinding proteins highly expressed on surfaces of cells [48]. It regulates cell-to-cell cooperation, immunity, and extracellular interactions, playing a prominent role in inflammation, coagulation, thrombosis, and malignancy [35]. Recent studies have shown that Gal-3 might be an independent biomarker of vascular remodeling and endothelial dysfunction accompanied with inflammation, proliferation, and atherosclerosis in the general population as well as in diabetic patients [35, 41]. Specifically in diabetic patients, Gal-3 was proposed to be a predictor of coronary sclerosis [40]. Amongst individuals with established CV diseases, increases in serum Gal-3 are associated 
with higher risk of heart failure (HF), T2DM, and CV mortality rate $[40,41]$. Specifically in patients with HF, Gal-3 is still not established as a trusted prognostic marker of cardiovascular outcome [42].

Additionally, Gal-3 has not demonstrated to be superior to other markers such as CRP, NPs, soluble suppression of tumorigenicity 2 (ST2) or growth differentiation factor 15 (GDF15 ) as a predictor of vasculopathy, low kidney function, and CV mortality in patients with T2DM [43]. Nevertheless, there is a large body of evidence suggesting that Gal-3 might be a target of the contemporary treatment for patients with T2DM [41].

Cardiac troponins are biomarkers of myocardial injury, which may exhibit high predictive value for CV death in subjects with established coronary artery disease, myocardial infarction, acute coronary syndrome, and HF [43]. Nevertheless, cardiac troponins may appear independently associated with a higher CV mortality risk in the general population, including obese and T2DM subpopulations [43, 44]. Mildly elevated levels of highsensitivity circulating troponin T (hs-cTnT) in untreated T2DM patients were recently found to be an independent predictor of death beyond traditional CV risk factors, excluding myocardial infarction and HF [45]. Furthermore, elevated level of troponins in diabetic patients do not always precede primary cardiacrelated diseases, but they have frequently been associated with pulmonary thromboembolism, atrial fibrillation, and thromboembolic complications including stroke [46]. Long-term monitoring of hs-cTnT might be useful to stratify diabetics at higher risk of all-cause mortality, even though their predictive accuracy may be suboptimal with other co-morbidities present [47]. Large clinical trials are required to determine whether cardiac troponins correspond to the natural evolution of diabetic cardiomyopathy along all the stages.

\section{Perspectives}

Current knowledge allows us to assert established patterns of biomarker behavior in different stages of glucose dysregulation. However, elucidating in which direction should future research be guided to is still in progress. To elucidate the clinical utility of these temporary relationships, we must overcome several limitations.

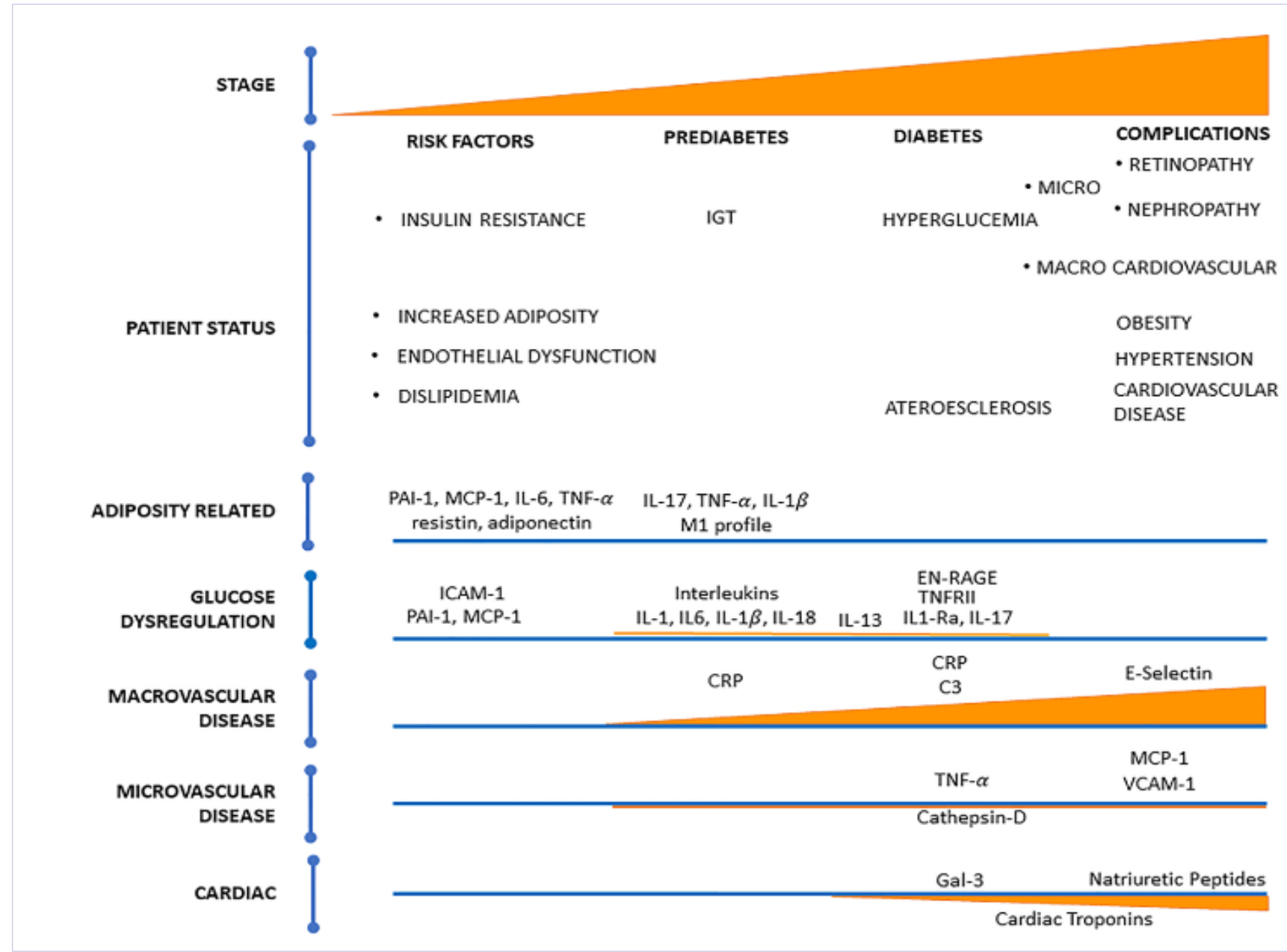

Figure 1: Timeline on biomarkers reported at different stages of T2DM development. Several biomarkers can be found from incipient stages of metabolic dysregulation produced by risk factors to late stages of diabetic complications, present either from the beginning of T2DM or to arising at specific stages. Certain T2DM biomarkers of complications have been reported for diagnostic purposes or as a risk assessment indicator. 
Reports on biomarker behavior were often discordant. Related factors include the use of different measurement techniques for the same marker and the heterogeneity of each population. This allows for limited generalization of the meaningfulness of each finding. The perspective of biomarker use also varies from risk predictors to diagnostic tests of established conditions. An exemplifying case is the use of hs-cTnT as an indicator of higher mortality risk in diabetics or IL-13 as an indicator of progression from normoglycemia to pre-diabetes. Additionally, the presence of some biomarkers is heterogeneous, appearing in diseases that range from cancer to diabetes, as is the case of IL-17A or ENRAGE, limiting their specificity.

Future efforts should be oriented to enlightening the relationship between IL-13, IL-17 and EN-RAGE, leading to the creation of a metabolic profile to be tested as a risk-stratifying tool. Also, serial measurements of hs-cTnT exclusively in diabetics with a long follow-up period would confirm the association previously found in patients free of heart disease.

We summarize the phase specific behavior of the most relevant biomarkers discussed in this review (see Figure 1).

\section{Conclusion}

Evidence shown about the phase specific behavior of biomarkers in the stages of T2DM provided us with viable biomarker profiles to be postulate as instruments for risk stratifying or diagnostic purposes. The existence of such an instrument represents a powerful asset that could decrease disease burden.

Future efforts should aim to reach agreement for the employment of biomarkers profiles to serve as assistance for clinicians in their daily practice.

\section{Acknowledgements}

We thank Manuel Hernández and Guillermo Luna for their technical help.

\section{Sources of Support}

Supported by Programa de Apoyo a Proyectos de Investigación e Innovación Tecnológica (PAPIIT, www.dgapa.unam.mx/html/ papiit/papit.html) IN218016 and IV100116, and Consejo Nacional de Ciencia y Tecnología (CONACyT, www.conacyt.gob. $\mathrm{mx}) 255635$ granted to DEG.

\section{References}

1. Weber K, Nowotny B, Strassburger K, Pacini G, Müssig K, Szendroedi J, et al. The Role of Markers of Low-Grade Inflammation for the Early Time Course of Glycemic Control, Glucose Disappearance Rate, and $\beta$-Cell Function in Recently Diagnosed Type 1 and Type 2 Diabetes. Diabetes Care. 2015;38(9):1758-1767. Doi: 10.2337/dc15-0169

2. Brahimaj A, Ligthart S, Ikram M, Hofman A, Franco O, Sijbrands E, et al. Serum Levels of Apolipoproteins and Incident Type 2 Diabetes: A Prospective Cohort Study. Diabetes Care. 2016;40(3):346-351. Doi: 10.2337/dc16-1295
3. Brahimaj A, Ligthart S, Ghanbari M, Ikram M, Hofman A, Franco O, et al. Novel inflammatory markers for incident pre-diabetes and type 2 diabetes: the Rotterdam Study. European Journal of Epidemiology. 2017;32(3):217-226. Doi: 10.1007/s10654-017-0236-0

4. Visser M, Bouter LM, McQuillan GM, Wener MH, Harris TB. Elevated C-Reactive Protein Levels in Overweight and Obese Adults. JAMA. 1999;282(22):2131-2135.

5. Stepien M, Stepien A, Wlazel R, Paradowski M, Banach M, Rysz J. Obesity indices and inflammatory markers in obese non-diabetic normo- And hypertensive patients. a comparative pilot study. Atherosclerosis. 2014;235(2):e136-e137. Doi: 10.1186/1476-511X-13-29

6. Matsuzawa Y. Adiponectin: Identification, physiology and clinical relevance in metabolic and vascular disease. Atherosclerosis Supplements. 2005;6(2):7-14. Doi: 10.1016/j. atherosclerosissup.2005.02.003

7. Herder C, Baumert J, Thorand B, Koenig W, de Jager W, Meisinger C et al. Chemokines as risk factors for type 2 diabetes: results from the MONICA/KORA Augsburg study, 1984-2002. Diabetologia. 2006;49(5):921-929. Doi: 10.1007/s00125-006-0190-y

8. Pirola L, Ferraz J. Role of pro- and anti-inflammatory phenomena in the physiopathology of type 2 diabetes and obesity. World Journal of Biological Chemistry. 2017;8(2):120-128. Doi: 10.4331/wjbc. v8.i2.120

9. Beringer A, Noack M, Miossec P. IL-17 in Chronic Inflammation: From Discovery to Targeting. Trends in Molecular Medicine. 2016;22(3):230241. Doi: 10.1016/j.molmed.2016.01.001

10. Chen C, Shao Y. Elevated Interleukin-17 Levels in Patients with Newly Diagnosed Type 2 Diabetes Mellitus. Biochemistry \& Physiology: Open Access. 2016;5(2).

11. Afzal N, Javaid K, Sami W, Zafar A, Jahan S, Zaman S, et al. Inverse Relationship of Serum IL-17 with Type-II Diabetes Retinopathy. Clinical Laboratory. 2013;59(11-12)1311-1317.

12. Guilherme A, Virbasius J, Puri V, Czech M. Adipocyte dysfunctions linking obesity to insulin resistance and type 2 diabetes. Nature Reviews Molecular Cell Biology. 2008;9(5):367-377. Doi: 10.1038/ nrm2391

13. Reddy S, Amutha A, Rajalakshmi R, Bhaskaran R, Monickaraj F, Rangasamy $S$ et al. Association of increased levels of MCP-1 and cathepsin-D in young onset type 2 diabetes patients (T2DM-Y) with severity of diabetic retinopathy. Journal of Diabetes and its Complications. 2017;31(5):804-809. Doi: 10.1016/j. jdiacomp.2017.02.017

14. Goldberg R. Cytokine and Cytokine-Like Inflammation Markers, Endothelial Dysfunction, and Imbalanced Coagulation in Development of Diabetes and Its Complications. The Journal of Clinical Endocrinology \& Metabolism. 2009;94(9):3171-3182. Doi: 10.1210/jc.2008-2534

15. Libby P, Ridker P. Inflammation and atherosclerosis: role of C-Reactive protein in risk assessment. The American Journal of Medicine. 2004;116(6):9-16. Doi: 10.1016/j.amjmed.2004.02.006

16. Derosa G, Maffioli P. A review about biomarkers for the investigation of vascular function and impairment in diabetes mellitus. Vascular Health and Risk Management. 2016;12:415-419. Doi: 10.2147/VHRM. S64460 
17. Schölin A, Siegbahn A, Lind L, Berne C, Sundkvist G, Björk E et al. CRP and IL- 6 concentrations are associated with poor glycemic control despite preserved $\beta$-cell function during the first year after diagnosis of type 1 diabetes. Diabetes/Metabolism Research and Reviews. 2004;20(3):205-210. Doi: 10.1002/dmrr.427

18. Pradhan A, Manson JE, Rifai N, Buring JE, Ridker PM. C-Reactive Protein, Interleukin 6, and Risk of Developing Type 2 Diabetes Mellitus. JAMA. 2001;286(3):327-334.

19. Bhowmick K, Kutty A, Shetty H. Glycemic control modifies the association between microalbuminuria and c-reactive protein in Type 2 Diabetes Mellitus. Indian Journal of Clinical Biochemistry. 2007;22(2):53-59. Doi: 10.1007/BF02913314

20. Müller S, Martin S, Koenig W, Hanifi-Moghaddam P, Rathmann W, Haastert B, et al. Impaired glucose tolerance is associated with increased serum concentrations of interleukin 6 and co-regulated acute-phase proteins but not TNF- $\alpha$ or its receptors. Diabetologia. 2002;45(6):805-812. Doi: 10.1007/s00125-002-0829-2

21. Ballak D, Stienstra R, Tack C, Dinarello C, van Diepen J. IL-1 family members in the pathogenesis and treatment of metabolic disease: Focus on adipose tissue inflammation and insulin resistance. Cytokine. 2015;75(2):280-290. Doi: 10.1016/j.cyto.2015.05.005

22. Kulkarni H, Mamtani M, Peralta J, Almeida M, Dyer T, Goring $\mathrm{H}$ et al. Soluble Forms of Intercellular and Vascular Cell Adhesion Molecules Independently Predict Progression to Type 2 Diabetes in Mexican American Families. PLOS ONE. 2016;11(3):e0151177. Doi: 10.1371/ journal.pone. 0151177

23. Mosmann T, Coffman R. Two types of mouse helper T-cell clone. Immunology Today. 1987;8(7-8):223-227.

24. Stanya K, Jacobi D, Liu S, Bhargava P, Dai L, Gangl M et al. Direct control of hepatic glucose production by interleukin-13 in mice. Journal of Clinical Investigation. 2012;123(1):261-271. Doi: 10.1172/JCI64941

25. Sumarac-Dumanovic M, Jeremic D, Pantovic A, Janjetovic $K$, Stamenkovic-Pejkovic D, Cvijovic G et al. Therapeutic improvement of glucoregulation in newly diagnosed type 2 diabetes patients is associated with a reduction of IL-17 levels. Immunobiology. 2013;218(8):1113-1118. Doi: 10.1016/j.imbio.2013.03.002

26. Pan A, Wang Y, Yuan J, Koh W. High-sensitive C-reactive protein and risk of incident type 2 diabetes: a case-control study nested within the Singapore Chinese Health Study. BMC Endocrine Disorders. 2017;17(1):8. Doi: 10.1186/s12902-017-0159-5

27. de Jager J, Dekker JM, Kooy A, Kostense PJ, Nijpels G, Heine RJ, et al. Endothelial Dysfunction and Low-Grade Inflammation Explain Much of the Excess Cardiovascular Mortality in Individuals With Type 2 Diabetes: The Hoorn Study. Arteriosclerosis, Thrombosis, and Vascular Biology. 2006;26(5):1086-1093. Doi: 10.1161/01. ATV.0000215951.36219.a4

28. Chen Y, Qiao Y, Xu Y, Ling W, Pan Y, Huang Y et al. Serum TNF- $\alpha$ concentrations in type 2 diabetes mellitus patients and diabetic nephropathy patients: A systematic review and metaanalysis. Immunology Letters. 2017;186:52-58. Doi: 10.1016/j. imlet.2017.04.003

29. Luis-Rodríguez D. Pathophysiological role and therapeutic implications of inflammation in diabetic nephropathy. World Journal of Diabetes. 2012;3(1):7-18. Doi: 10.4239/wjd.v3.i1.7
30. Hocaoglu-Emre F, Saribal D, Yenmis G, Guvenen G. Vascular Cell Adhesion Molecule 1, Intercellular Adhesion Molecule 1, and Cluster of Differentiation 146 Levels in Patients with Type 2 Diabetes with Complications. Endocrinology and Metabolism. 2017;32(1):99-105. Doi: 10.3803/EnM.2017.32.1.99

31. Liu J, Yeoh L, Sum C, Tavintharan S, Ng X, Liu S et al. Vascular cell adhesion molecule-1, but not intercellular adhesion molecule-1, is associated with diabetic kidney disease in Asians with type 2 diabetes. Journal of Diabetes and its Complications. 2015;29(5):707-712. Doi: 10.1016/j.jdiacomp.2015.02.011

32. Kato K, Osawa H, Ochi M, Kusunoki Y, Ebisui O, Ohno K et al. Serum total and high molecular weight adiponectin levels are correlated with the severity of diabetic retinopathy and nephropathy. Clinical Endocrinology. 2008;68(3):442-449. Doi: 10.1111/j.13652265.2007.03063.x

33. Ersanli D, Top C, Öncül O, Aydin A, Terekeci H. Relationship between serum soluble E-selectin levels and development of diabetic retinopathy in patients with type 2 diabetes. Scandinavian Journal of Clinical and Laboratory Investigation. 2007;67(5):474-479. Doi: 10.1080/00365510601110148

34. Nowak C, Sundström J, Gustafsson S, Giedraitis V, Lind L, Ingelsson E et al. Protein biomarkers for insulin resistance and type 2 diabetes risk in two large community cohorts. Diabetes. 2016;65(1):276-284. Doi : $10.2337 / \mathrm{db} 15-0881$

35. Berezin A. The Rationality to Use of Galectin-3 as Target in BiomarkerGuided Therapy of Type 2 Diabetes Mellitus. Endocrinology \& Metabolic Syndrome. 2016;05(01).

36. Moro C. Targeting cardiac natriuretic peptides in the therapy of diabetes and obesity. Expert Opinion on Therapeutic Targets. 2016;20(12):1445-1452. Doi: 10.1080/14728222.2016.1254198

37. E Berezin A. Cardiovascular Biomarkers in Routine Screening of Diabetic Patients. Clinical \& Medical Biochemistry: Open Access. 2015;01(01).

38. Correction to: 2016 ACC/AHA/HFSA Focused Update on New Pharmacological Therapy for Heart Failure: An Update of the 2013 ACCF/AHA Guideline for the Management of Heart Failure: A Report of the American College of Cardiology Foundation/American Heart Association Task Force on Clinical Practice Guidelines and the Heart Failure Society of America. Circulation. 2016;134(13):e298-e298. Doi: 10.1161/CIR.0000000000000435

39. Seferović P. ESC/HFA Guidelines for the Diagnosis and Treatment of Acute and Chronic Heart Failure 2016. Journal of Cardiac Failure. 2017;23(10):S7. Doi: 10.1016/j.cardfail.2017.08.005

40. Ozturk D, Celik O, Satilmis S, Aslan S, Erturk M, Cakmak H et al. Association between serum galectin-3 levels and coronary atherosclerosis and plaque burden/structure in patients with type 2 diabetes mellitus. Coronary Artery Disease. 2015;26(5):396-401. Doi: 10.1097/MCA.0000000000000252

41. Shah R, Januzzi J. Soluble ST2 and Galectin-3 in Heart Failure. Clinics in Laboratory Medicine. 2014;34(1):87-97. Doi: 10.1016/j. cll.2013.11.009

42. Coburn E, Frishman W. Comprehensive Review of the Prognostic Value of Galectin-3 in Heart Failure. Cardiology in Review. 2014;22(4):171175. Doi: $10.1097 /$ CRD.0000000000000013 
43. Alonso N, Lupón J, Barallat J, de Antonio M, Domingo M, Zamora E et al. Impact of diabetes on the predictive value of heart failure biomarkers. Cardiovascular Diabetology. 2016;15(1):151. Doi: 10.1186/s12933016-0470-x

44. Scirica B, Bhatt D, Braunwald E, Raz I, Cavender M, Im K et al Prognostic Implications of Biomarker Assessments in Patients With Type 2 Diabetes at High Cardiovascular Risk. JAMA Cardiology. 2016;1(9):989-998. Doi: 10.1001/jamacardio.2016.3030
45. Pareek M, Nielsen M, Leósdóttir M, Nilsson P, Olsen M. 4B.07. Journal of Hypertension. 2015;33:e54-e55.

46. Akoum N. New perspectives on atrial fibrillation and stroke. Heart. 2016;102(22):1788-1792. Doi: 10.1136/heartjnl-2015-309066

47. McEvoy J, Lazo M, Chen Y, Shen L, Nambi V, Hoogeveen R et al. Patterns and determinants of temporal change in high-sensitivity cardiac troponin-T: The Atherosclerosis Risk in Communities Cohort Study. International Journal of Cardiology. 2015;187:651-657. Doi: 10.1016/j.ijcard.2015.03.436 